\title{
DLTS Investigations of (Ga,In)(N,As)/GaAs Quantum Wells before and after Rapid Thermal Annealing
}

\author{
Ł. GelczuK*, M. DAzBrowska-Szata And D. Pucicki \\ Faculty of Microsystem Electronics and Photonics, Wrocław University of Technology, \\ Z. Janiszewskiego 11/17, 50-372 Wrocław, Poland
}

\begin{abstract}
Deep level transient spectroscopy was used to investigate deep-level defects in (Ga,In)(N,As)/GaAs triple quantum well structures grown by atmospheric pressure metalorganic vapor phase epitaxy with different indium and nitrogen contents and annealed in rapid thermal annealing system. A combination of electron traps that disappear or remain on annealing and a new hole trap that appears on annealing were detected. The revealed electron traps were attributed to N-related complexes or GaAs host-related native point defects. Moreover, it was suggested that the new hole trap observed in the annealed GaAsN/GaAs triple quantum well structure together with the dominant electron trap can act as generation-recombination center responsible for the observed a very poor optical quality among all the investigated multi-quantum well structures.
\end{abstract}

DOI: $10.12693 /$ APhysPolA.126.1195

PACS: 71.55.Eq, 71.55.-i, 73.61.Ey

\section{Introduction}

The $(\mathrm{Ga}, \mathrm{In})(\mathrm{N}, \mathrm{As})$ semiconductor alloys, so-called dilute nitrides are currently of great interest due to their unique properties such as a huge and negative band gap bowing coefficient and large conduction band offset, which result from a large size and electronegativity difference between $\mathrm{N}$ and As atoms. These features make III-V-N alloys very promising material for applications in 1.3-1.55 $\mu \mathrm{m}$ telecommunication lasers [1] and photodetectors [2], as well as high efficient multi-junction solar cells [3]. The introduction of small amounts of nitrogen into GaAs considerably decreases band gap energy of GaNAs down to $\approx 1 \mathrm{eV}$ and simultaneously reduces the lattice parameters of the crystal. Moreover, incorporation of indium to GaNAs additionally compensates the mechanical strains and improve crystal quality, which could be lattice matched to common substrates such as GaAs or Ge. Unfortunately, a small amount of nitrogen strongly deteriorates the material quality of diluted nitrides due to a high concentration of impurities (carbon, hydrogen, oxygen) and native point defects (vacancies, interstitials, antisities), which can be the centers of non-radiative recombination and cause low luminescence efficiency or influence on poor minority-carrier diffusion lengths, their mobilities and lifetimes. Therefore, the fundamental goal is to correlate the properties of defects with the electrical and optical properties of $(\mathrm{Ga}, \mathrm{In})(\mathrm{N}, \mathrm{As})$ alloys.

The purpose of this paper is to present the investigations of deep-level defects by means of deep level transient spectroscopy (DLTS) method [4] in (Ga,In)(N,As)/GaAs

${ }^{*}$ corresponding author; e-mail: lukasz.gelczuk@pwr.edu.pl multi-quantum well (MQW) structures grown by atmospheric pressure metalorganic vapor phase epitaxy (APMOVPE) technique. Both as-grown and annealed structures were investigated. On the basis of the obtained electrical parameters of the traps and literature reports, their possible origin was proposed.

\section{Experiment}

The investigated (Ga,In)(N,As)/GaAs heterostructures were grown by APMOVPE with AIX200 R\&D AIXTRON horizontal reactor on (100)-oriented Si-doped $\left(\approx 10^{18} \mathrm{~cm}^{-3}\right)$ GaAs substrates. The growth precursors were trimethylgallium (TMGa), trimethylindium (TMIn), tertiarybutylhydrazine (TBHy) and $10 \%$ mixture of $\mathrm{AsH}_{3}$ in $\mathrm{H}_{2}$, and they were transported by passing a high purity $\mathrm{H}_{2}$ through the bubblers. Undoped MQW structures consisted of $500 \mathrm{~nm}$ thick buffer and $3 \times(\mathrm{Ga}, \mathrm{In})(\mathrm{N}, \mathrm{As})$ quantum wells, separated by $30 \mathrm{~nm}$ of GaAs barrier and capped by $45 \mathrm{~nm}$ of GaAs. In the experiment, three different structures containing MQW were used: GaInAs/GaAs (sample I219n), GaNAs/GaAs (sample N62n) and GaInNAs/GaAs (sample NI45n). All the structures were grown at the same temperature equal to $575^{\circ} \mathrm{C}$. The main growth parameters were reported elsewhere [5]. The In and $\mathrm{N}$ contents in QWs were determined by combination of high resolution X-ray diffraction (HRXRD) and contactless electroreflectance (CER) measurements, assisted by band anticrossing (BAC) calculations, according to the algorithm proposed by Pucicki et al. [6]. In this approach, comparison of the results achieved from simulated HRXRD curves with the calculations of all QWs transitions measured by CER leads to reduction of deviations in composition determination of GaInNAs QWs and thereby to increasing the accuracy in determining of the In and $\mathrm{N}$ contents. 
After the growth process, the samples were annealed for 5 min at $700{ }^{\circ} \mathrm{C}$ under $\mathrm{N}_{2}$ atmosphere in rapid thermal annealing (RTA) system, in order to investigate the influence of thermal treating on the evolution and parameters of deep-level traps.

For DLTS measurements, circular Au Schottky contacts $\left(0.5 \mathrm{~mm}^{2}\right)$ were deposited on topside of the samples and AuGe ohmic contacts on the whole backside. The measurement setup consists of 2601A Keithley SMU instrument, Boonton 7200 capacitance bridge, Lakeshore 331 temperature controller and Janis VPF-475 liquid nitrogen cryostat.

\section{Results and discussion}

Figure 1 shows the DLTS spectra obtained for the three investigated as-grown and annealed $(\mathrm{Ga}, \mathrm{In})(\mathrm{N}, \mathrm{As}) / \mathrm{GaAs}$ $3 \times$ QWs structures. The DLTS measurements were performed within the $80-480 \mathrm{~K}$ temperature range, with a reverse bias voltage $\left(U_{\mathrm{R}}\right)$ of $-2 \mathrm{~V}$, a filling pulse voltage $\left(U_{\mathrm{P}}\right)$ of $0 \mathrm{~V}$, an emission rate window $\left(e_{n}\right)$ of $20 \mathrm{~s}^{-1}$, and a filling pulse width $\left(t_{\mathrm{p}}\right)$ of $1 \mathrm{~ms}$. The positive DLTS signal corresponds to majority electron traps, labeled as $\mathrm{E}$ and the negative one is associated with minority hole traps, labeled as $\mathrm{H}$.

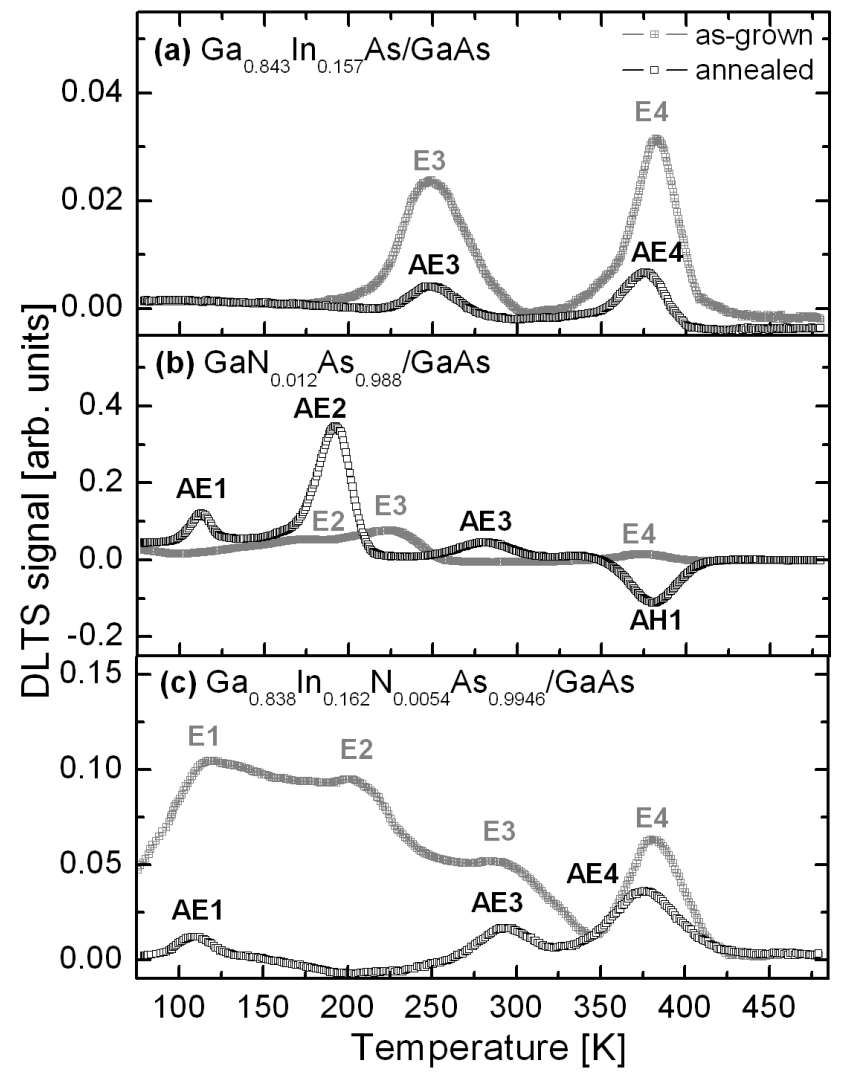

Fig. 1. Standard DLTS spectra obtained for asgrown (gray lines) and annealed (black lines) $(\mathrm{Ga}, \mathrm{In})(\mathrm{N}, \mathrm{As}) / \mathrm{GaAs} 3 \times \mathrm{QWs}$ structures of various nitrogen and indium contents.
As it is shown in Fig. 1, within the measured temperature range DLTS spectra reveal a presence of two (E3, E4), three (E2, E3, E4), or four (E1, E2, E3, E4) deep electron traps in the as-grown GaInAs/GaAs, GaNAs/GaAs and GaInNAs/GaAs structures, respectively. After the post-growth RTA $\left(700^{\circ} \mathrm{C}, 5 \mathrm{~min}\right)$ some of the traps disappear completely but also some of them appear and the others keep almost resistant upon annealing or their concentration slightly decreases. In effect, two (AE3, AE4), three (AE1, AE2, AE3), and three (AE1, AE3, AE4), electron traps are revealed in the annealed samples, respectively. Furthermore, an additional hole trap, labeled AH1, has emerged from the DLTS spectrum of annealed GaNAs/GaAs structure at a higher temperature, instead of electron trap E4. The existence of hole trap in this sample can be related to higher density of carbon contaminations among all the investigated structures.

In this work, the TBHy was used as a source of nitrogen in the growth of dilute GaNAs and GaInNAs quantum wells, which could be also the additional source of carbon contamination. The investigated samples were grown without intentional doping, but they actually exhibited low background carrier concentrations at about $\approx 10^{16} \mathrm{~cm}^{3}$ after the growth process, which decreased slightly after RTA. It is generally known that carbon can be incorporated during growth of diluted nitrides at sufficiently high levels causing probably the background $p$-type conductivity [3]. Therefore, the highest nitrogen concentration in the GaNAs QWs (of about 1.2\%) can be also connected with higher carbon contamination, giving rise to $p$-type conductivity in annealed GaNAs QWs.

Moreover, one can see in Fig. 1 that some of the DLTS spectra are distinctly broadened (e.g. DLTS spectrum for the as-grown GaInNAs/GaAs structure). A broad DLTS-line suggests that deep levels, giving rise to this signal, can be in fact a continuous distribution of deep level states or groups of closely spaced discrete energy levels within the band gap. In order to resolve the closely spaced defect levels, high-resolution Laplace DLTS (LDLTS) was applied [7].

The basic electrical parameters of the revealed traps, i.e. thermal activation energy $\left(E_{\mathrm{a}}=E_{\mathrm{C}, \mathrm{V}} \pm E_{\mathrm{T}}\right)$ and apparent capture cross-section $\left(\sigma_{\mathrm{a}}\right)$ were determined from the slope and intersection values of the Arrhenius plots shown in Fig. 2, respectively. The concentration of each trap $\left(N_{\mathrm{T}}\right)$ was determined from the DLTS-peak height. The calculated activation energies and capture crosssections (assumed to be temperature independent in this study), as well as concentrations of the revealed traps are collected in Table for the as-grown and annealed $(\mathrm{Ga}, \mathrm{In})(\mathrm{N}, \mathrm{As}) / \mathrm{GaAs}$ structures, respectively.

On the basis of the obtained results and the data reported in the literature for the deep-level traps observed in dilute GaNAs and GaInNAs alloys we tried to identify the possible origins of the traps in the investigated samples. The first electron trap E1 (labeled AE1 in the annealed samples) that is not observed 


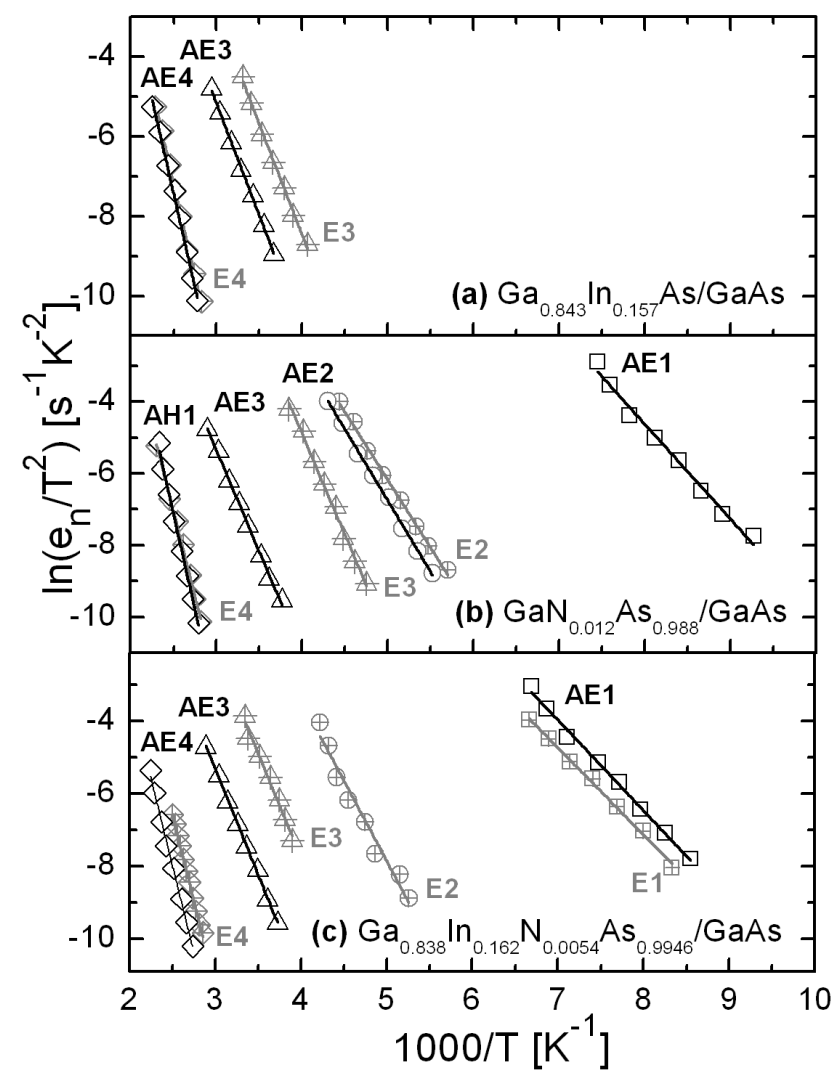

Fig. 2. The Arrhenius plots of DLTS spectra given in Fig. 1 obtained by standard DLTS and highresolution LDLTS measurements of thermal emission rates.

TABLE

Summary of activation energy $\left(E_{\mathrm{a}}\right)$, apparent capture cross-section $\left(\sigma_{\mathrm{a}}\right)$ and concentration $\left(N_{\mathrm{T}}\right)$ of the deep-level traps obtained from standard DLTS and Laplace DLTS analysis of as-grown and annealed $(\mathrm{Ga}, \mathrm{In})(\mathrm{N}, \mathrm{As}) / \mathrm{GaAs}$ quantum well structures.

\begin{tabular}{|c|c|c|c|c|c|}
\hline Sample & Treatment & $\begin{array}{l}\text { Trap } \\
\text { label }\end{array}$ & $\begin{array}{c}E_{\mathrm{a}} \\
{[\mathrm{eV}]}\end{array}$ & $\begin{array}{c}\sigma_{\mathrm{a}} \\
{\left[\mathrm{cm}^{2}\right]}\end{array}$ & $\begin{array}{c}N_{\mathrm{T}} \\
{\left[\mathrm{cm}^{-3}\right]}\end{array}$ \\
\hline \multirow{4}{*}{$\begin{array}{c}\mathrm{I} 219 \mathrm{n} \\
\text { GaInAs }\end{array}$} & \multirow[t]{2}{*}{ as-grown } & E3 & 0.479 & $1.57 \times 10^{-15}$ & $7.1 \times 10^{12}$ \\
\hline & & $\mathrm{E} 4$ & 0.807 & $1.86 \times 10^{-14}$ & $9.2 \times 10^{12}$ \\
\hline & \multirow[t]{2}{*}{ annealed } & AE3 & 0.484 & $1.44 \times 10^{-15}$ & $2.5 \times 10^{12}$ \\
\hline & & $\mathrm{AE} 4$ & 0.812 & $1.69 \times 10^{-14}$ & $3.1 \times 10^{12}$ \\
\hline \multirow{7}{*}{$\begin{array}{c}\text { N62n } \\
\text { GaNAs }\end{array}$} & \multirow[t]{3}{*}{ as-grown } & E2 & 0.326 & $5.57 \times 10^{-15}$ & $9.3 \times 10^{12}$ \\
\hline & & E3 & 0.485 & $7.30 \times 10^{-14}$ & $1.3 \times 10^{13}$ \\
\hline & & E4 & 0.815 & $2.58 \times 10^{-14}$ & $2.4 \times 10^{12}$ \\
\hline & \multirow[t]{4}{*}{ annealed } & AE1 & 0.228 & $2.49 \times 10^{-14}$ & $2.7 \times 10^{13}$ \\
\hline & & AE2 & 0.342 & $8.02 \times 10^{-15}$ & $7.5 \times 10^{13}$ \\
\hline & & AE3 & 0.487 & $1.82 \times 10^{-15}$ & $9.2 \times 10^{12}$ \\
\hline & & AH1 & 0.857 & $8.03 \times 10^{-15}$ & $2.2 \times 10^{13}$ \\
\hline \multirow{7}{*}{$\begin{array}{c}\text { NI45n } \\
\text { GaInNAs }\end{array}$} & \multirow[t]{4}{*}{ as-grown } & E1 & 0.207 & $1.08 \times 10^{-16}$ & $3.1 \times 10^{13}$ \\
\hline & & $\mathrm{E} 2$ & 0.382 & $1.03 \times 10^{-15}$ & $2.7 \times 10^{13}$ \\
\hline & & E3 & 0.495 & $6.56 \times 10^{-14}$ & $1.4 \times 10^{13}$ \\
\hline & & E4 & 0.816 & $4.41 \times 10^{-14}$ & $1.5 \times 10^{13}$ \\
\hline & \multirow[t]{3}{*}{ annealed } & AE1 & 0.215 & $1.21 \times 10^{-15}$ & $2.3 \times 10^{12}$ \\
\hline & & AE3 & 0.494 & $2.15 \times 10^{-15}$ & $3.0 \times 10^{12}$ \\
\hline & & $\mathrm{AE} 4$ & 0.818 & $1.49 \times 10^{-14}$ & $6.4 \times 10^{12}$ \\
\hline
\end{tabular}

in the GaInAs/GaAs structure should involve N atoms. This trap appears only after annealing of GaNAs/GaAs structure, while it is present in both as-grown and annealed GaInNAs/GaAs structure, respectively. After annealing of the latter sample the concentration of this trap clearly decreases. Finally, after annealing the concentration of this trap is about one order of magnitude higher for the GaNAs/GaAs structure than for GaInNAs/GaAs one, which can be related to higher N content in GaNAs QWs. According to theoretical predictions, N complexes, such as nitrogen split interstitials $\mathrm{N}-\mathrm{As}$ and $\mathrm{N}-\mathrm{N}$ on single As sites (i.e. $\mathrm{N}_{\mathrm{i}}-\mathrm{As}_{\mathrm{As}}$ and $\mathrm{N}_{\mathrm{i}}-\mathrm{N}_{\mathrm{As}}$ ) are energetically favorable in dilute nitride alloys [8]. Experimental data confirm that as-grown GaNAs layers contain a significant concentration of nitrogen interstitials [9]. Furthermore, it is also known that these complexes are partially responsible for compensation of the tensile and compressive strain in $(\mathrm{Ga}, \mathrm{In})(\mathrm{N}, \mathrm{As})$ layers grown on GaAs substrate. Thus, they can lead to significant deviation of the lattice parameter values from the Vegard law [10] and low luminescence efficiency [9]. On the basis of these findings, we can identify the trap E1 (AE1) as being due to such N-complexes. Particularly, we tentatively attributed this trap to $\mathrm{N}_{\mathrm{i}}-\mathrm{As}_{\mathrm{As}}$ defect, because experimental results confirm that this defect usually persists both isochronal and isothermal annealing, due to its higher formation energy compared to $\mathrm{N}_{\mathrm{i}}-\mathrm{N}_{\mathrm{As}}$, which are instead strongly reduced after annealing [11].

The electron trap E2 (AE2) is also observed only in the dilute GaAsN/GaAs and GaInNAs/GaAs heterostructures. Furthermore, after annealing the concentration of this trap strongly increases in the former sample but it completely disappears in the latter one. Nevertheless, this trap manifests parameters comparable to the wellknown EL6 defect, which is commonly observed in a bulk and epitaxial GaAs and GaAsN [12], being regarded as a possible candidate of the effective recombination center.

On the other hand, the electron trap E3 (AE3) is observed in all the investigated as-grown and annealed samples. However, the concentration of this trap decreases strongly after annealing. According to the obtained electrical parameters of this trap presented in Table, it can be attributed to another well-known defect usually observed in GaAs and GaAs-based alloys, called EL3. It is suggested that a defect responsible for the EL3 trap, due to its close proximity to the midgap, is also regarded as a possible candidate for major non-radiative recombination center in $(\mathrm{Ga}, \mathrm{In})(\mathrm{N}, \mathrm{As})$ layers [12].

The last electron trap E4 (AE4) is also observed in all the investigated $(\mathrm{Ga}, \mathrm{In})(\mathrm{N}, \mathrm{As}) / \mathrm{GaAs}$ structures after the growth process. Nevertheless, the concentration of this trap strongly decreases in the GaInAs/GaAs and GaInNAs/GaAs structures after RTA, while it completely disappears in the GaNAs/GaAs structure. Instead of the trap E4, a new hole trap AH1 appears in the DLTS spectrum of annealed GaNAs/GaAs structure at a higher temperature. The electron trap E4 (AE4) can be also associated with another native point defect, called EL2, 
which is one of the most important and widely studied defect commonly observed in GaAs and GaAs-based alloys [13].

As it was mentioned above, the presence of the minority hole trap $\mathrm{AH} 1$ in the unintentionally doped GaNAs/GaAs structures, is probably related to enhanced carbon incorporation during the growth process of the dilute nitride QWs that can be responsible to the $p$-type conductivity of these layers. A similar deep hole trap labeled H1 was previously observed by Kaplar et al. [14] in MOCVD grown Sn-doped GaInNAs/GaAs heterostructures after annealing. They proposed the relationship of the hole trap $\mathrm{H} 1\left(E_{\mathrm{V}}+0.71 \mathrm{eV}\right)$ with another deep electron trap E2 $\left(E_{\mathrm{C}}-0.36 \mathrm{eV}\right)$, observed by them in the annealed samples. Furthermore, they found that the activation energies of these two traps just add to $1.07 \mathrm{eV}$, that is very close to the $1.05 \mathrm{eV}$ band gap of the GaInNAs material studied by them. Basing on this findings, they concluded that E2 and $\mathrm{H} 1$ may be the same defect, acting as both the electron and hole trap and thus being the efficient generation-recombination center (G-R center). Recently, we have also revealed similar E2/H1 defect in the as-grown GaInNAs/GaAs triple quantum well structures, grown by APMOVPE at distinctly lower temperature of $566^{\circ} \mathrm{C}[15]$. In that paper, the sum of activation energies of the two traps was equal to $1.19 \mathrm{eV}$, that is also very close to about $1.2 \mathrm{eV}$ band gap of GaInNAs measured by CER.

In the present paper, the sum of activation energies of the electron trap AE2 $\left(E_{\mathrm{C}}-0.34 \mathrm{eV}\right)$ and the hole trap $\mathrm{AH} 1\left(E_{\mathrm{V}}+0.86 \mathrm{eV}\right)$ is equal to $1.2 \mathrm{eV}$, that is also close to the $1.22 \mathrm{eV}$ bandgap energy of the annealed GaNAs/GaAs structure, as measured by photoluminescence (PL) [5]. Moreover, the concentration of these two traps strongly increases after annealing, being the dominant deep-level defects in this structure. On the basis of the given findings we can attribute the AE2/AH1 to G-R center presents in GaNAs/GaAs $3 \times$ QWs structure. Thus, it is probably the main deep-level defect responsible for a very poor optical quality observed in GaNAs/GaAs MQWs, revealed recently by means of the $\mathrm{PL}$ and CER investigations [5].

\section{Conclusion}

We have presented the DLTS investigation of $(\mathrm{Ga}, \mathrm{In})(\mathrm{N}, \mathrm{As}) / \mathrm{GaAs}$ triple QW structures grown at the same temperature by APMOVPE and annealed under nitrogen atmosphere. On the basis of the DLTS results, the deep electron trap E1 (AE1) observed only in the dilute nitride structures was attributed to N-related complexes (probably $\mathrm{N}_{\mathrm{i}}-\mathrm{As}_{\mathrm{As}}$ defect). On the other hand, the electron traps E2 (AE2), E3 (AE3) and E4 (AE4) were associated with the common native GaAs host-related defects called EL6, EL3 and EL2, respectively. Finally, the deep hole trap AH1, observed only in the GaNAs/GaAs structure after annealing and the electron trap AE2 were proposed to be the same deep-level defect. The sum of activation energies of the $\mathrm{AH} 1$ and $\mathrm{AE} 2$ traps is very close to the bandgap energy of the $\mathrm{GaN}_{0.012} \mathrm{As}_{0.988}$ alloy. Thus, the trap AE2/AH1 can act as the efficient generation-recombination center, being responsible for a very poor optical quality of the GaNAs/GaAs $3 \times$ QWs structure measured recently by means of PL and CER.

\section{Acknowledgments}

This work was co-financed by Polish Ministry of Science and Higher Education under the grant no. N N507 552938. The authors are grateful to the group of Prof. Marek Tłaczała (Wrocław University of Technology) for growing the investigated structures.

\section{References}

[1] M. Fischer, D. Gollub, M. Reinhardt, M. Kamp, A. Forchel, J. Cryst. Growth 251, 353 (2003).

[2] Q. Han, X.H. Yang, Z.C. Niu, H.Q. Ni, Y.Q. Xu, S.Y. Zhang, Y. Du, L.H. Peng, Appl. Phys. Lett. 87, 111105 (2005).

[3] J.F. Geisz, D.J. Friedman, Semicond. Sci. Technol. 17, 769 (2002).

[4] D.V. Lang, J. Appl. Phys. 45, 3023 (1974).

[5] W. Dawidowski, B. Ściana, M. Latkowska, D. Radziewicz, D. Pucicki, K. Bielak, M. Badura, M. Tłaczała, Proc. SPIE 8902, 89022G (2013).

[6] D. Pucicki, K. Bielak, R. Kudrawiec, D. Radziewicz, B. Ściana, Mater. Sci.-Poland 31, 489 (2013).

[7] L. Dobaczewski, A.R. Peaker, K. Bonde Nielsen, J. Appl. Phys. 96, 4689 (2004).

[8] S.B. Zhang, S.-H. Wei, Phys. Rev. Lett. 86, 1789 (2001).

[9] W. Li, M. Pessa, T. Ahlgren, J. Dekker, Appl. Phys. Lett. 79, 1094 (2001).

[10] W. Li, M. Pessa, J. Likonen, Appl. Phys. Lett. 78, 2864 (2001).

[11] P. Krispin, V. Gambin, J.S. Harris, K.H. Ploog, J. Appl. Phys. 93, 6095 (2003).

[12] A.Y. Polyakov, N.B. Smirnov, A.V. Govorkov, A.E. Botchkarev, N.N. Nelson, M.M.E. Fahmi, J.A. Griffin, A. Khan, S.N. Mohammad, D.K. Johnstone, V.T. Bublik, K.D. Chsherbatchev, M.I. Voronova, V.S. Kasatochkin, Solid State Electron. 46, 2155 (2002).

[13] M. Kamińska, E.R. Weber, in: Imperfections in III/V Materials, Semiconductors and Semimetals, Eds. E.R. Weber, Vol. 8, Academic Press, Boston 1993, p. 59.

[14] R.J. Kaplar, A.R. Arehart, S.A. Ringel, A.A. Allerman, R.M. Sieg, S.R. Kurtz, J. Appl. Phys. 90, 3405 (2001).

[15] Ł. Gelczuk, M. Dąbrowska-Szata, P. Kamyczek, E. Płaczek-Popko, K. Kopalko, B. Ściana, D. Pucicki, D. Radziewicz, M. Tłaczała, Proc. SPIE 8902, 89020F (2013). 